\title{
Editorial
}

\section{Can an intramucosal undifferentiated-type gastric cancer become a candidate for endoscopic submucosal resection?}

\author{
Seigo Kitano \\ Department of Surgery I, Oita University Faculty of Medicine, 1-1 Idaigaoka, Yufu, Oita 879-5593, Japan
}

Recently in Japan, the incidence of early gastric cancer (EGC) has increased to more than $50 \%$ of all gastric cancers with the development of diagnostic modalities and mass examination. The rate of lymph node metastasis in EGC is known to be very low: about $2 \%-5 \%$ in intramucosal cancer and $15 \%-25 \%$ in submucosal cancer [1]. When there is no risk of lymph node metastasis, EGC can be completely removed by endoscopic treatment. The distribution of lymph node metastasis in early gastric cancer has been examined for the last 20 years with the purpose of clarifying the types of EGC with no risk of lymph node metastasis. Based on these studies, the guidelines for endoscopic treatment conducted by the Japanese Gastric Cancer Association recommend that empirical indications for endoscopic mucosal resection (EMR) be considered as papillary or tubular (differentiated) adenocarcinoma less than $2 \mathrm{~cm}$ in diameter without ulceration and lymphatic or vessel involvement [2]. This indication for EMR is widely accepted in Japan; however, many experienced endoscopists will consider this indication to be excessively strict. For the complete resection of ECG by endoscopic treatment, the importance of en-bloc resection has been emphasized for the prevention of local recurrence. With the development of endoscopic submucosal resection (ESD), en-bloc resection of large tumors has been achieved [3]. Therefore, the following expanded criteria for endoscopic treatment have been proposed: (1) differentiated mucosal cancer without ulceration, (2) differentiated mucosal cancer less than $3 \mathrm{~cm}$ in diameter with ulceration, (3) differentiated submucosal (SM1) cancer less than $3 \mathrm{~cm}$ in diameter, or (4) undifferentiated mucosal cancer (MC) less than $2 \mathrm{~cm}$ in diameter without lymphatic or vessel involvement [4].

In the expanded criteria, the most discussed area is the undifferentiated MC. There are few studies on the

Offprint requests to: S. Kitano distribution of lymph node metastasis in undifferentiated MC. Abe et al. reported that undifferentiated MC less than $10 \mathrm{~mm}$ in diameter without lymphatic or vessel involvement has no risk of lymph node metastasis [5]. Park et al. showed that the incidence of lymph node metastasis was very low in undifferentiated MC and undifferentiated submucosal $(<500 \mu \mathrm{m})$ cancer [6]. Hirasawa et al. showed in this journal that there was no risk of lymph node metastasis in undifferentiated MC less than $20 \mathrm{~mm}$ in diameter without ulceration and lymphatic or vessel involvement, and proposed that such types of undifferentiated MC might be treated by ESD [7]. The Hirasawa study analyzed a large amount of data from high-volume centers and seems to be both reliable and informative. Undifferentiated MC that meets these criteria may be a candidate for ESD in the near future.

There are several problems, however, if one is to completely accept Hirasawa's criteria of ESD for undifferentiated MC in the clinical setting. The study was performed based on the analysis of pathologic findings of resected specimens after surgery. The measurement of tumor size under endoscopic view is not always the same as when using the resected specimen. The diagnosis of the area of undifferentiated MC by endoscopy is also more difficult than that of differentiated $\mathrm{MC}$ because individual cancer cells of undifferentiated MC may separately infiltrate the gastric wall and lymphatic vessels. Similarly, the accuracy of pre-treatment diagnosis for cancer depth in undifferentiated MC is lower than that in differentiated MC. Thus, for the treatment of undifferentiated MC by ESD, development of pretreatment diagnosis for the size, the area, and the depth of the lesion may be necessary. It may be possible to regard ESD as a total biopsy for undifferentiated MC; the necessity of additional gastrectomy with lymph node dissection is decided according to histological examination of the specimen resected by ESD. The methods to evaluate the resected specimen after endoscopic treat- 
ment have been developed using differentiated MC after EMR. Using this evaluation system to evaluate the undifferentiated MC specimen resected after ESD may be neither feasible nor warranted. Furthermore, there is the risk of activating the growth and progression of the remnant undifferentiated cancer cells after ESD. Although Hirasawa's criteria of ESD for undifferentiated $\mathrm{MC}$ are useful and informative, evaluation methods of resected specimens after ESD for undifferentiated MC have to be developed.

ESD increases the rate of en-bloc resection of EGC and the number of cases with complete resection of large EGC. However, there is a risk of increasing the incidence of complications, especially bleeding and perforation associated with ESD. The review article by Gotoda et al. shows that the incidences of bleeding and perforation were $1.7 \%-38 \%$ and $0 \%-5 \%$, respectively [3]. The ESD study group in Korea showed that the incidence of delayed bleeding after ESD was higher in the middle and upper stomach than in the lower stomach [8]. Additionally, the delayed bleeding frequently occurred after ESD for EGC of more than $40 \mathrm{~mm}$ in diameter. Because undifferentiated MC is often located in the middle stomach, it is possible that delayed bleeding after ESD might be increased. Kakuhima et al. showed the existence of a learning curve in the acquisition of safe ESD techniques and demonstrated that a decrease in procedure time was found to be a marker of proficiency in this technique [9]. If undifferentiated MC less than $2 \mathrm{~cm}$ in diameter without lymphatic or vessel involvement were added to the indication for ESD as per Hirosawa's criteria, only laparoscopists with advanced techniques should attempt the resection of the lesion by ESD. This would be necessary for the prevention of delayed bleeding.

Although empirical indications for endoscopic treatment have been accepted in Japan, the expanded criteria have not yet been accepted because of the lack of supportive clinical evidence. Hirasawa's study published in this journal [7] supports the indication of undifferentiated MC as shown in the expanded criteria. In order to accept undifferentiated $\mathrm{MC}$ as a candidate for ESD, improvement of pre-treatment diagnosis and evaluation of resected specimens by ESD is necessary. Furthermore, training and technical education in ESD techniques are required for the prevention of complications. Finally, clinical evidence of the oncologic and technical feasibility of ESD for undifferentiated MC must be presented in the near future.

\section{References}

1. Maruyama K, Kaminishi M, Hayashi K, Isobe Y, Honda I, Katai H, et al. Gastric cancer treated in 1991 in Japan: data analysis of nationwide registry. Gastric Cancer 2006;9:51-66.

2. Japanese Gastric Cancer Association. Gastric cancer treatment guidelines. 2nd ed. (in Japanese) Tokyo: Kanahara; 2004.

3. Gotoda T, Yamamoto H, Soetikno RM. Endoscopic submucosal dissection of early gastric cancer. J Gastroenterol 2006;41:929-42.

4. Gotoda T, Yanagisawa A, Sasako M, Ono H, Nakanishi Y, Shimoda $\mathrm{T}$, et al. Incidence of lymph node metastasis from early gastric cancer: estimation with a large number of cases at two large centers. Gastric Cancer 2000;3:219-25.

5. Abe N, Watanabe T, Sugiyama M, Yanagida O, Masaki T, Mori $\mathrm{T}$, et al. Endoscopic treatment or surgery for undifferentiated early gastric cancer? Am J Surg 2004;188:181-4.

6. Park YD, Chung YJ, Chung HY, Yu W, Bae HI, Jeon SW, et al. Factors related to lymph node metastasis and the feasibility of endoscopic mucosal resection for treating poorly differentiated adenocarcinoma of the stomach. Endoscopy 2008;40:7-10.

7. Hirasawa T, Gotoda T, Miyata S, Kato Y, Shimoda T, Taniguchi $\mathrm{H}$, et al. Incidence of lymph node metastasis and the feasibility of endoscopic resection for undifferentiated-type early gastric cancer. Gastric Cancer 2009;12:148-52.

8. Chung IK, Lee JH, Lee SH, Kim SJ, Cho JY, Cho WY, et al. Therapeutic outcome in 1000 cases of endoscopic submucosal dissection for early gastric neoplasm: Korean ESD Study Group multicenter study. Gastrointest Endosc 2009;69:1228-35.

9. Kakuhima M, Fujishiro M, Kodashima S, Muraki Y, Tateishi A, Omata M. A learning curve for endoscopic submucosal dissection of gastric epithelial neoplasms. Endoscopy 2006;38:991-5. 